\title{
IAMJ
}

INTERNATIONAL AYURVEDIC MEDICAL JOURNAL

ISSN: $2320-5091$

Impact Factor: 6.719

\section{THE MANAGEMENT OF AMAVATA (RHEUMATOID ARTHRITIS) WITH VATARI GUGGULU AND BRIHAT SIMHANADA GUGGULU}

\author{
Praveen Kumar $^{1}$, Sriram Chandra Mishra ${ }^{2}$, Vandana Gupta ${ }^{3}$ \\ ${ }^{1}$ PG Scholar, ${ }^{2}$ Professor \& H.O.D., ${ }^{3}$ Associate Professor., \\ Department of Kayachikitsa, Vaidya Yagya Dutt Sharma Ayurveda Mahavidyalaya, \\ Khurja, Bulandshahar (U.P.) 203131, India
}

Corresponding Author: dr.praveengangwar@gmail.com

https://doi.org/10.46607/iamj0209022021

(Published online: February 2021)

Open Access

(C) International Ayurvedic Medical Journal, India 2021

Article Received: 14/01/2021 - Peer Reviewed: 23/01/2021 - Accepted for Publication: 24/01/2021

\section{D) Check for updates}

\section{ABSTRACT}

The detail knowledge on Amavata was first explained by Madhavakar, whereas Chakrapani Dutta first gave knowledge about principle and management of the disease. Amavata is a clinical entity very much similar to the chronic but active inflammatory arthropathy, the Rheumatoid arthritis. Till now, the etio-pathogenesis of Rheumatoid arthritis is not known precisely but among the hypothesis, entero-pathy along with autoimmune have important role regarding this disease. In Amavata, due to impaired functioning of 'Kayagni' the anna-rasa undergoes fermentation resulted formation of ama (biotoxin) which combines with vitiated Vata (biophysical force for movement) to form Amavata.(1) So, two important entities one is toxin and other is movement, when comes together kha vaigunya concept the disease formed which is worst one. That's why swelling, severe pain, and restricted movements are the main features of Amavata. Severe pain, difficulty in movements, and swelling on the joints along with fever etc makes the patient's life miserable. Although Ama and Vata are chiefly pathogenic factors, Kapha and Pitta are also invariably involved in its pathogenesis (Samprapti). The therapeutic approach should be on Vata dosha, Kapha dosha and correction of Amadosha and of Agni viz. Pitta. The line of treatment for amavata also includes langhanam, swedanam, tiktam, deepana, katu drugs and sodhana treatment like virechana, basti etc. The shamana drugs which are having Vatashamaka, Amapachaka, Ama Shoshaka, and Deepniya properties can be used in the treatment of this disease. Vatari Guggulu and Brihat simhanada guggulu 
carries indication for Amavata according to Bhaisajya Ratnavali. The compositions in it are approachable lieu of principles of treatment of Amavata. The clinical research shows that in TG I, 7 (46.67\%) patients were got Moderate improvement while 8 (53.33\%) patients were got Mild improvement. In TG II 10 (66.67\%) patients were get Moderate improvement while 5 (33.33\%) patients were got Mild improvement.

Keyword- Amavata, Vatari Guggulu, Rheumatoid arthritis, Brihat simhanada guggulu and Ayurveda.

\section{INTRODUCTION}

Ayurveda maintains the "Swasthya of swastha (healthy) person and cures the vikaras of an atura (diseased) person. The samprapti of almost all the diseases begins with the Agnimandya "Rogah sarve api mandeagnou". Agnimandya leads to formation of Ama. This Ama is the most important pathological factor responsible for the various diseases. One of the most challenging diseases caused by Ama is Amavata. Amavata is defined as a condition where Ama and Vata Dosha are vitiated simultaneously and enters the Trika (Pelvic girdle) and Sandhi (joints) causing stiffness (Stabdhata) of the body. Here, Ama means improperly/partially digested food or undigested Rasa Dhatu formed due to poor strength of Agni. It simultaneously affects the joints and causes stiffness of the body. Pain and inflammation of one or more joints particularly of hand, foot, tarsals, knee, thigh and sacrum; scorpion bite like pain in the affected joints, loss of appetite, indigestion; fever, vague pain in different parts of the body, feeling of heaviness in the body, lack of initiative, disturbance in sleep habit, stiffness in chest (heart area), constipation are the signs and symptoms of Amavata. The disease ultimately leads to the disability.

Amavata displays many features in common with a collection of signs and symptoms that are typically diagnosed as "Rheumatoid Arthritis" which is a chronic autoimmune disease that causes inflammation and deformity of the joints.(2) RA can also cause inflammation of the tissues around the joints as well as other organs in the body. It is a common disorder, with varied clinical signs and symptoms related to multiple anatomical sites, both articular and extraarticular. It is a chronic progressive disease-causing inflammation in the joints and resulting in painful de- formity and immobility, especially in the fingers, wrists, feet and ankles.

RA is widely prevalent throughout the world. According to the annual European Congress of Rheumatology (EULAR 2018 report), Rheumatoid arthritis (RA) is the most common autoimmune inflammatory form of arthritis and affects approximately 1 in 100 people worldwide, with women affected twice as commonly as men.(3)

According to the association of physicians of India, RA steadily increases to $5 \%$ in women over the age of 70. Rheumatoid arthritis (RA) affects about $0.92 \%$ of adult population in India. There are about 20-40 new cases per Lac population each year and the disease occurs more frequently in females. (4)

In Ayurveda, the line of treatment for amavata also includes langhanam, swedanam, tiktam, deepana, katu drugs and sodhana treatment like virechana, basti etc.(5) The therapeutic approach should be on Vata dosha, Kapha dosha and correction of Amadosha and of Agni viz. Pitta. The drugs which are having Vatashamaka, Amapachaka, Ama Shoshaka, and Deepniya properties can be used in the treatment of this disease. There are various medications elaborated for samana therapy in amavata. Among them, two drugs (Vatari Guggulu and Brihat Simhanada Guggulu) had been selected for clinical evaluation on the management of Amavata (Rheumatoid arthritis) Joint degeneration has become a commonplace problem in aging populations. The main clinical manifestations include joint pain, joint stiffness and joint swelling with functional disorder. Mega MSM is a nutritional supplement that may provide potential relief for joint problems associated with joint degeneration. The current experiment performed was a 12-week, randomized, double-blind, controlled study conducted on 
populations in China experiencing joint degeneration. The objective of the study was to determine whether the daily use of Mega MSM capsules could improve joint function, relieve symptoms of joint degeneration and improve the quality of life in aging populations. A total of 100 male and female participants over 50 years old who had at least one of the related symptoms of joint degeneration (joint pain, joint stiffness, joint swelling, difficulty walking, difficulty getting up from bed and difficulty going down stairs) were recruited and their symptoms of joint degeneration before and after the intervention were recorded. In this study, Mega MSM shows positive effects in improving joint function, relieving symptoms associated with joint degeneration and improving the quality of life in aging populations (4). Vatari Guggulu carries indication for Amavata, katisula, gridhrasi, khanja, vatarakta, pangu, sotha, daha etc. Brihat Simhanada guggulu is a known virechaka drug also carries indication for Amavata, sandhivata, katigraha, medo-kapha-ama samghata etc.(5) The compositions in both the drugs are approachable lieu of principles of treatment of Amavata. Simhanada Guggulu and Vatari Guggulu have Guggulu as main ingredient. Both formulations are used as anti-inflammatory drugs. This effect is probably due to guggulsterones present in both drugs. Guggulsterone is reported to inhibit significantly lipopolysaccharide-induced upregulation of tumor necrosis factor-alpha expression and cyclooxygenase-2 production. (4) Most of the ingredients of above formulations are Ushna Virya, Katu Vipaka, and having Snigdha and Ruksha properties. Ushna Virya drugs are helpful in digesting and absorbing the vitiated Ama by its Shoshana Karma. The Deepniya property of these drugs also slows down the Ama formation. Hence both drugs are selected for the study undertaken with the title "A clinical comparative study on the management of Amavata (Rheumatoid arthritis) with Vatari Guggulu and Brihat Simhanada Guggulu”.

\section{Observation and Results}

Total 30 patients were considered. They were divided into two groups $\left(\mathrm{TG}_{\mathrm{I}}, \mathrm{TG}_{\mathrm{II}}\right)$, each group having 15 patients.

\section{Observation on Clinical Data}

1. Showing the incidence of patients according to Presence of clinical features

\begin{tabular}{|c|c|c|c|c|c|c|}
\hline \multirow[t]{2}{*}{ Sl. No } & \multirow{2}{*}{\multicolumn{2}{|c|}{ Clinical Features }} & \multicolumn{2}{|c|}{ TG I } & \multicolumn{2}{|c|}{ TG II } \\
\hline & & & $f$ & $\%$ & $f$ & $\%$ \\
\hline 1 & \multicolumn{2}{|c|}{ Stabdha gatrata (Morning stiffness) } & 15 & $100 \%$ & 15 & $100 \%$ \\
\hline 2 & \multicolumn{2}{|c|}{ Angamarda (Bodyache) } & 15 & $100 \%$ & 15 & $100 \%$ \\
\hline 3 & \multicolumn{2}{|c|}{ Aruchi (Anorexia) } & 11 & $73.33 \%$ & 10 & $66.67 \%$ \\
\hline 4 & \multicolumn{2}{|l|}{ Trishna (Thirst) } & 6 & $40 \%$ & 11 & $73.33 \%$ \\
\hline 5 & \multicolumn{2}{|c|}{ Alasya (Laziness) } & 8 & $53.33 \%$ & 9 & $60 \%$ \\
\hline 6 & \multicolumn{2}{|c|}{ Gaurava (Heaviness) } & 15 & $100 \%$ & 15 & $100 \%$ \\
\hline 7 & \multicolumn{2}{|c|}{ Apaka (Indigestion) } & 15 & $100 \%$ & 15 & $100 \%$ \\
\hline 8 & \multicolumn{2}{|c|}{ Sunatanganam (Sandhi sotha/swelling in joints) } & 15 & $100 \%$ & 15 & $100 \%$ \\
\hline 9 & \multicolumn{2}{|c|}{ Ruja (Continuous pain in joints like Scorpion bites) } & 15 & $100 \%$ & 15 & $100 \%$ \\
\hline 10 & \multicolumn{2}{|l|}{ Jwara (Fever) } & 6 & $40 \%$ & 8 & $53.33 \%$ \\
\hline \multirow[t]{2}{*}{11} & \multirow[t]{2}{*}{ Grip strength } & Right & 15 & $100 \%$ & 15 & $100 \%$ \\
\hline & & Left & 15 & $100 \%$ & 15 & $100 \%$ \\
\hline \multirow[t]{2}{*}{12} & \multirow[t]{2}{*}{ Foot pressure } & Right & 15 & $100 \%$ & 15 & $100 \%$ \\
\hline & & Left & 15 & $100 \%$ & 15 & $100 \%$ \\
\hline 13 & \multicolumn{2}{|c|}{ Rheumatoid Arthritis score (ACR / EULAR -2010) } & 15 & $100 \%$ & 15 & $100 \%$ \\
\hline 14 & \multicolumn{2}{|c|}{ Simple disease activity index score } & 15 & $100 \%$ & 15 & $100 \%$ \\
\hline
\end{tabular}

Observation - In this study, presence of clinical features shows 15 (100\%) in Stabdha gatrata (Morning stiffness), Angamarda (Bodyache), Gaurava (Heavi- ness), Apaka (Indigestion), Sunatanganam (Sandhi Sotha/swelling in joints), Ruja (Continuous pain in joints like Scorpion bites). $11 \quad(73.33 \%)$ \& 10 
(66.67\%) in Aruchi (Anorexia), 6 (40\%) \& 11 (73.33\%) in Trishna (Thirst), 8 (53.33\%) \& $9(60 \%)$ in Alasya (Laziness), 6 (40\%) \& $8(53.33 \%)$ in Jwara (fever) of TG I \&TG II respectively. In both groups,
Grip strength (Right), Grip strength (Left), Foot pressure (Right), Foot pressure (Left), Rheumatoid Arthritis score (ACR / EULAR -2010), Simple disease activity index score was assessed in all patients.

2. Showing the incidence of patients according to Degree of Severity of sign \& symptoms in subjective parameters Before and After treatment in TG I

\begin{tabular}{|c|c|c|c|c|c|}
\hline \multirow[t]{2}{*}{ Sign \& Symptoms } & \multirow[t]{2}{*}{ Gradation } & \multicolumn{2}{|c|}{ TG I } & \multicolumn{2}{|c|}{ TG II } \\
\hline & & BT & $\mathbf{A T}$ & BT & AT \\
\hline \multirow[t]{4}{*}{ Stabdha gatrata (Morning stiffness) } & G0 & 0 & 1 & 0 & 3 \\
\hline & G1 & 0 & 11 & 1 & 10 \\
\hline & G2 & 9 & 3 & 10 & 2 \\
\hline & G3 & 6 & 0 & 4 & 0 \\
\hline \multirow{4}{*}{ Angamarda (Bodyache) } & G0 & 0 & 3 & 0 & 2 \\
\hline & G1 & 0 & 5 & 1 & 10 \\
\hline & G2 & 8 & 7 & 6 & 3 \\
\hline & G3 & 7 & 0 & 8 & 0 \\
\hline \multirow{4}{*}{ Aruchi (Anorexia) } & G0 & 0 & 5 & 0 & 4 \\
\hline & G1 & 1 & 4 & 1 & 5 \\
\hline & G2 & 10 & 2 & 8 & 1 \\
\hline & G3 & 0 & 0 & 1 & 0 \\
\hline \multirow{4}{*}{ Trishna (Thirst) } & G0 & 0 & 1 & 0 & 3 \\
\hline & G1 & 1 & 4 & 2 & 5 \\
\hline & G2 & 3 & 1 & 3 & 3 \\
\hline & G3 & 2 & 0 & 6 & 0 \\
\hline \multirow[t]{4}{*}{ Alasya (Laziness) } & G0 & 0 & 1 & 0 & 2 \\
\hline & G1 & 2 & 5 & 2 & 5 \\
\hline & $\mathrm{G} 2$ & 5 & 2 & 6 & 2 \\
\hline & G3 & 1 & 0 & 1 & 0 \\
\hline \multirow{4}{*}{ Gaurava (Heaviness) } & G0 & 0 & 3 & 0 & 4 \\
\hline & G1 & 2 & 9 & 2 & 8 \\
\hline & $\mathrm{G} 2$ & 8 & 3 & 8 & 3 \\
\hline & G3 & 5 & 0 & 5 & 0 \\
\hline \multirow{4}{*}{ Apaka (Indigestion) } & G0 & 0 & 2 & 0 & 5 \\
\hline & G1 & 0 & 10 & 2 & 7 \\
\hline & G2 & 9 & 3 & 9 & 3 \\
\hline & G3 & 6 & 0 & 4 & 0 \\
\hline \multirow[t]{4}{*}{ Sunatanganam (Sandhi sotha/swelling in joints) } & G0 & 0 & 3 & 0 & 4 \\
\hline & G1 & 0 & 9 & 1 & 7 \\
\hline & $\mathrm{G} 2$ & 11 & 3 & 8 & 4 \\
\hline & G3 & 4 & 0 & 6 & 0 \\
\hline \multirow[t]{4}{*}{ Ruja (Continuous pain in joints like Scorpion bites) } & G0 & 0 & 3 & 0 & 3 \\
\hline & G1 & 1 & 6 & 1 & 7 \\
\hline & G2 & 7 & 6 & 6 & 5 \\
\hline & G3 & 7 & 0 & 8 & 0 \\
\hline \multirow[t]{4}{*}{ Jwara (Fever) } & G0 & 0 & 7 & 0 & 7 \\
\hline & G1 & 6 & 1 & 6 & 1 \\
\hline & $\mathrm{G} 2$ & 2 & 0 & 2 & 0 \\
\hline & G3 & 0 & 0 & 0 & 0 \\
\hline \multirow[t]{3}{*}{ Grip strength (RIGHT) } & G0 & 0 & 1 & 0 & 1 \\
\hline & G1 & 0 & 11 & 0 & 11 \\
\hline & G2 & 9 & 3 & 9 & 3 \\
\hline
\end{tabular}




\begin{tabular}{|l|l|l|l|l|l|}
\hline & G3 & 6 & 0 & 6 & 0 \\
\hline Grip strength (LEFT) & G0 & 0 & 1 & 0 & 1 \\
\hline Foot pressure (RIGHT) & G1 & 0 & 11 & 0 & 11 \\
\hline Foot pressure (LEFT) & G2 & 9 & 3 & 9 & 3 \\
\hline & G3 & 6 & 0 & 6 & 0 \\
\hline Rheumatoid Arthritis score (ACR / EULAR -2010) & G1 & 0 & 1 & 0 & 1 \\
\hline Simple disease activity index score & G2 & 0 & 11 & 0 & 11 \\
\hline & G3 & 9 & 3 & 9 & 3 \\
\hline & G0 & 0 & 0 & 6 & 0 \\
\hline & G1 & 0 & 1 & 0 & 1 \\
\hline & G3 & 9 & 3 & 9 & 11 \\
\hline & G0 & 6 & 0 & 6 & 0 \\
\hline & G1 & 0 & 1 & 0 & 3 \\
\hline & G2 & 0 & 11 & 0 & 10 \\
\hline
\end{tabular}

\section{Observation}

Regarding Individual features like Stabdha Gatrata (Morning stiffness), TGI shows $0,0,9,6$ cases of before treatment came to $1,11,3,0$ after treatment whereas TGII shows $0,1,10,4$ cases of before treatment came to $3,10,2,0$ after treatment in respected severity gradations of $\mathrm{G}_{0}, \mathrm{G}_{1}, \mathrm{G}_{2}, \mathrm{G}_{3}$.
Regarding Individual features like Angamarda (Body ache), TGI shows $0,0,8,7$ cases of before treatment came to 3,5, 7, 0 after treatment whereas TGII shows $0,1,6,8$ cases of before treatment came to $2,10,3,0$ after treatment in respected severity gradations of $\mathrm{G}_{0}$, $\mathrm{G}_{1}, \mathrm{G}_{2}, \mathrm{G}_{3}$.

3. Statistical analysis showing the effectiveness of Trial drugs in both groups

\begin{tabular}{|c|c|c|c|c|c|c|c|c|c|}
\hline \multicolumn{2}{|l|}{ Sign \& Symptoms } & \multicolumn{2}{|c|}{ Mean Score } & \multirow{2}{*}{$\begin{array}{l}\text { Mean } \\
\text { diff. }\end{array}$} & \multirow{2}{*}{$\begin{array}{l} \pm \\
\text { S.D. }\end{array}$} & \multirow{2}{*}{$\begin{array}{l} \pm \\
\text { S.E. }\end{array}$} & \multirow{2}{*}{$\begin{array}{l}\text { w- } \\
\text { Value }\end{array}$} & \multirow{2}{*}{$\begin{array}{l}\text { p - Val- } \\
\text { ue }\end{array}$} & \multirow[t]{2}{*}{ Remark } \\
\hline & & B.T. & A.T. & & & & & & \\
\hline \multirow{2}{*}{$\begin{array}{l}\text { Stabdha gatrata (Morning stiff- } \\
\text { ness) }\end{array}$} & TG I & 2.40 & 1.13 & 1.27 & 0.46 & 0.12 & 120.00 & $<0.0001$ & E.S. \\
\hline & TG II & 2.20 & 0.93 & 1.27 & 0.46 & 0.12 & 120.00 & $<0.0001$ & E.S. \\
\hline \multirow[t]{2}{*}{ Angamarda (Bodyache) } & TG I & 2.47 & 1.27 & 1.20 & 0.68 & 0.17 & 91.00 & 0.0002 & E.S. \\
\hline & TG II & 2.47 & 1.07 & 1.40 & 0.51 & 0.13 & 120.00 & $<0.0001$ & E.S. \\
\hline \multirow[t]{2}{*}{ Aruchi (Anorexia) } & TG I & 1.91 & 0.73 & 1.18 & 0.75 & 0.23 & 45.00 & 0.0039 & V.S. \\
\hline & TG II & 2.00 & 0.70 & 1.30 & 0.48 & 0.15 & 55.00 & 0.002 & V.S. \\
\hline \multirow[t]{2}{*}{ Trishna (Thirst) } & TG I & 2.17 & 1.00 & 1.17 & 0.41 & 0.17 & 21.00 & 0.0313 & S. \\
\hline & TG II & 2.36 & 1.00 & 1.36 & 0.50 & 0.15 & 66.00 & 0.001 & E.S. \\
\hline \multirow[t]{2}{*}{ Alasya (Laziness) } & TG I & 1.88 & 1.13 & 0.75 & 0.46 & 0.16 & 21.00 & 0.0313 & S. \\
\hline & TG II & 1.89 & 1.00 & 0.89 & 1.05 & 0.35 & 15.00 & 0.0625 & N.Q.S. \\
\hline \multirow[t]{2}{*}{ Gaurava (Heaviness) } & TG I & 2.20 & 1.00 & 1.20 & 0.41 & 0.11 & 120.00 & $<0.0001$ & E.S. \\
\hline & TG II & 2.20 & 0.93 & 1.27 & 0.46 & 0.12 & 120.00 & $<0.0001$ & E.S. \\
\hline \multirow[t]{2}{*}{ Apaka (Indigestion) } & TG I & 2.40 & 1.07 & 1.33 & 0.62 & 0.16 & 105.00 & 0.0001 & E.S. \\
\hline & TG II & 2.13 & 0.87 & 1.27 & 0.46 & 0.12 & 120.00 & $<0.0001$ & E.S. \\
\hline \multirow{2}{*}{$\begin{array}{l}\text { Sunatanganam (Sandhi so- } \\
\text { tha/swelling in joints) }\end{array}$} & TG I & 2.27 & 1.00 & 1.27 & 0.59 & 0.15 & 120.00 & $<0.0001$ & E.S. \\
\hline & TG II & 2.33 & 1.00 & 1.33 & 0.49 & 0.13 & 120.00 & $<0.0001$ & E.S. \\
\hline \multirow{2}{*}{$\begin{array}{l}\text { Ruja (Continuous pain in joints } \\
\text { like Scorpion bites) }\end{array}$} & TG I & 2.40 & 1.20 & 1.20 & 0.41 & 0.11 & 120.00 & $<0.0001$ & E.S. \\
\hline & TG II & 2.47 & 1.13 & 1.33 & 0.49 & 0.13 & 120.00 & $<0.0001$ & E.S. \\
\hline
\end{tabular}




\begin{tabular}{|c|c|c|c|c|c|c|c|c|c|}
\hline \multicolumn{2}{|l|}{ Sign \& Symptoms } & \multicolumn{2}{|c|}{ Mean Score } & \multirow{3}{*}{$\begin{array}{l}\text { Mean } \\
\text { diff. }\end{array}$} & \multirow{3}{*}{$\begin{array}{l} \pm \\
\text { S.D. } \\
0.29\end{array}$} & \multirow{3}{*}{$\begin{array}{l} \pm \\
\text { S.E. } \\
0.12\end{array}$} & \multirow{3}{*}{$\begin{array}{l}\text { t- Val- } \\
\text { ue } \\
1137\end{array}$} & \multirow{3}{*}{$\begin{array}{l}\text { p - Val- } \\
\text { ue } \\
<00001\end{array}$} & \multirow{3}{*}{$\begin{array}{l}\text { Remark } \\
\text { E.S. }\end{array}$} \\
\hline & & B.T. & A.T. & & & & & & \\
\hline \multirow[t]{2}{*}{ Jwara (Fever) } & TG I & 99.65 & 98.42 & & & & & & \\
\hline & TG II & 99.81 & 98.40 & 1.41 & 0.38 & 0.13 & 10.524 & $<0.0001$ & E.S. \\
\hline \multirow[t]{2}{*}{ Grip strength (Right) } & TG I & 80.67 & 140.00 & 59.33 & 20.36 & 5.26 & 11.29 & $<0.0001$ & E.S. \\
\hline & TG II & 81.73 & 142.40 & -60.67 & 20.36 & 5.26 & 11.538 & $<0.0001$ & E.S. \\
\hline \multirow[t]{2}{*}{ Grip strength (Left) } & TG I & 82.13 & 142.67 & 60.53 & 29.69 & 7.67 & 7.90 & $<0.0001$ & E.S. \\
\hline & TG II & 83.73 & 146.67 & -62.93 & 32.83 & 8.48 & 7.43 & $<0.0001$ & E.S. \\
\hline \multirow[t]{2}{*}{ Foot pressure (Right) } & TG I & 12.73 & 22.00 & 9.27 & 4.30 & 1.11 & 8.35 & $<0.0001$ & E.S. \\
\hline & TG II & 12.67 & 22.13 & -9.47 & 4.44 & 1.15 & 8.26 & $<0.0001$ & E.S. \\
\hline \multirow[t]{2}{*}{ Foot pressure (Left) } & TG I & 12.60 & 21.60 & 9.00 & 4.47 & 1.16 & 7.79 & $<0.0001$ & E.S. \\
\hline & TG II & 12.93 & 22.27 & -9.33 & 4.32 & 1.12 & 8.37 & $<0.0001$ & E.S. \\
\hline \multirow{2}{*}{$\begin{array}{l}\text { Rheumatoid Arthritis score } \\
\text { (ACR / EULAR -2010) }\end{array}$} & TG I & 9.00 & 6.60 & 2.80 & 1.01 & 0.26 & 10.69 & $<0.0001$ & E.S. \\
\hline & TG II & 9.27 & 6.20 & 3.07 & 0.96 & 0.25 & 12.36 & $<0.0001$ & E.S. \\
\hline \multirow{2}{*}{$\begin{array}{l}\text { Simple disease activity index } \\
\text { score }\end{array}$} & TG I & 37.38 & 24.85 & 12.53 & 3.50 & 0.90 & 13.86 & $<0.0001$ & E.S. \\
\hline & TG II & 40.33 & 24.71 & 15.63 & 5.40 & 1.39 & 11.22 & $<0.0001$ & E.S. \\
\hline
\end{tabular}

4. Showing the incidence of patients according to the $\%$ of improvement after treatment in both group

\begin{tabular}{|l|l|l|}
\hline Cardinal features & \multicolumn{2}{l|}{$\%$ of improvement } \\
\cline { 2 - 2 } & TG I & TG II \\
\hline Stabdha gatrata (Morning stiffness) & 52.79 & 57.59 \\
\hline Angamarda (Bodyache) & 48.64 & 56.75 \\
\hline Aruchi (Anorexia) & 61.92 & 65.00 \\
\hline Trishna (Thirst) & 53.85 & 57.70 \\
\hline Alasya (Laziness) & 40.00 & 47.06 \\
\hline Gaurava (Heaviness) & 54.55 & 57.59 \\
\hline Apaka (Indigestion) & 55.54 & 59.40 \\
\hline Sunatanganam (Sandhi sotha/swelling in joints) & 55.89 & 57.14 \\
\hline Ruja (Continuous pain in joints like Scorpion bites) & 50.00 & 54.03 \\
\hline Jwara (Fever) & 1.35 & 1.42 \\
\hline Grip strength (Right) & 73.55 & 74.23 \\
\hline Grip strength (Left) & 73.70 \\
\hline Foot pressure (Right) & 72.78 & 75.16 \\
\hline Foot pressure (Left) & 71.43 \\
\hline Rheumatoid Arthritis score (ACR / EULAR -2010) & 31.11 \\
\hline Simple disease activity index score & 33.53 & 74.74 \\
\hline
\end{tabular}

\section{Observation}

Percentage (\%) of improvement among TG I \& TG II shows $52.79 \%$ \& $57.59 \%$ in Stabdha Gatrata (Morning stiffness), $48.64 \%$ \& $56.75 \%$ in Angamarda (Bodyache),61.92\% \& 65.00\% in Aruchi (Anorexia), $53.85 \%$ \& $57.70 \%$ in Trishna (Thirst), $40.00 \%$ \& $47.06 \%$ in Alasya (Laziness), $54.55 \%$ \& $57.59 \%$ in Gaurava (Heaviness), 55.54\% \& $59.40 \%$ in Apaka (Indigestion), $55.89 \%$ \& $57.14 \%$ in Sunatanganam
(Sandhi Sotha/swelling in joints), 50.00\% \& 54.03\% in Ruja (Continuous pain in joints like Scorpion bites), $1.35 \%$ \& $1.42 \%$ in Jwara (Fever), $73.55 \%$ \& $74.23 \%$ in Grip strength (Right), $73.70 \%$ \& $75.16 \%$ in Grip strength (Left), $72.78 \%$ \& $74.74 \%$ in Foot pressure (Right), 71.43\% \& 72.16\% in Foot pressure (Left), $31.11 \%$ \& $33.10 \%$ in Rheumatoid Arthritis score (ACR / EULAR -2010) , 33.53\% \& 38.74\% in Simple disease activity index score respectively. 
5. Comparison of effects on different parameters of both drugs

\begin{tabular}{|c|c|c|c|c|c|c|}
\hline \multicolumn{2}{|l|}{ Symptom } & \multirow{2}{*}{\begin{tabular}{|l} 
No of pts \\
15 \\
\end{tabular}} & \multirow{2}{*}{$\begin{array}{l}\text { Means } \\
1.267\end{array}$} & \multirow{2}{*}{$\begin{array}{l}\text { Mann-Whitney (U val- } \\
\text { ue) } \\
112.5\end{array}$} & \multirow{3}{*}{$\begin{array}{l}\text { p-Value } \\
0.9784\end{array}$} & \multirow{2}{*}{$\begin{array}{l}\text { Remark } \\
\text { not significant }\end{array}$} \\
\hline Stabdha gatrata (Morning stiffness) & TGI & & & & & \\
\hline & TGII & 15 & 1.267 & & & \\
\hline \multirow{2}{*}{ Angamarda (Bodyache) } & TGI & 15 & 1.2 & \multirow[t]{2}{*}{96} & \multirow[t]{2}{*}{0.4493} & \multirow{2}{*}{ not significant } \\
\hline & TGII & 15 & 1.4 & & & \\
\hline \multirow{2}{*}{ Aruchi (Anorexia) } & TGI & 11 & 1.182 & \multirow{2}{*}{51.5} & \multirow{2}{*}{0.8106} & \multirow{2}{*}{ not significant } \\
\hline & TGII & 10 & 1.3 & & & \\
\hline \multirow[t]{2}{*}{ Trishna (Thirst) } & TGI & 6 & 1.167 & \multirow[t]{2}{*}{26.5} & \multirow[t]{2}{*}{0.4456} & \multirow[t]{2}{*}{ not significant } \\
\hline & TGII & 11 & 1.364 & & & \\
\hline \multirow[t]{2}{*}{ Alasya (Laziness) } & TGI & 8 & 0.75 & \multirow[t]{2}{*}{35} & \multirow[t]{2}{*}{0.9574} & \multirow[t]{2}{*}{ not significant } \\
\hline & TGII & 9 & 0.8889 & & & \\
\hline \multirow[t]{2}{*}{ Gaurava (Heaviness) } & TGI & 15 & 1.2 & \multirow[t]{2}{*}{105} & \multirow[t]{2}{*}{0.692} & \multirow[t]{2}{*}{ not significant } \\
\hline & TGII & 15 & 1.267 & & & \\
\hline \multirow[t]{2}{*}{ Apaka (Indigestion) } & TGI & 15 & 1.333 & \multirow[t]{2}{*}{103} & \multirow[t]{2}{*}{0.6577} & not significant \\
\hline & TGII & 15 & 1.267 & & & \\
\hline Sunatanganam (Sandhi sotha) & TGI & 15 & 1.2 & 92.5 & 0.272 & not significant \\
\hline & TGII & 15 & 1.333 & & & \\
\hline Ruja (pain) & TGI & 15 & 1.2 & 97.5 & 0.4326 & not significant \\
\hline & TGII & 15 & 1.333 & & & \\
\hline Symptom & & No of pts & Means & t - value & p-Value & Remark \\
\hline Jwara (Fever) & TGI & 6 & 1.35 & 0.3618 & 0.7244 & not significant \\
\hline & TGII & 8 & 1.413 & & & \\
\hline Grip strength (Right) & TGI & 15 & 59.333 & 0.1793 & 0.859 & not significant \\
\hline & TGII & 15 & 60.667 & & & \\
\hline Grip strength (Left) & TGI & 15 & 60.533 & 0.21 & 0.8352 & not significant \\
\hline & TGII & 15 & 62.933 & & & \\
\hline Foot pressure (Right) & TGI & 15 & 9.267 & 0.1253 & 0.9012 & not significant \\
\hline & TGII & 15 & 9.467 & & & \\
\hline Foot pressure (Left) & TGI & 15 & 9 & 0.2076 & 0.8371 & not significant \\
\hline & TGII & 15 & 9.333 & & & \\
\hline Rheumatoid Arthritis score & TGI & 15 & 2.8 & 0.7391 & 0.4662 & not significant \\
\hline & TGII & 15 & 3.067 & & & \\
\hline SDAI score & TGI & 15 & 12.533 & 1.862 & 0.0749 & not significant \\
\hline & TGII & 15 & 15.627 & & & \\
\hline
\end{tabular}

Observation - The difference of effect of trial drug on various symptoms in TGI and TGII was not significant that means the relief provided by both group was nearly similar.

6. Showing the incidence of patients according to clinical effect of therapy

\begin{tabular}{|l|l|l|l|l|}
\hline Clinical effect of therapy & \multicolumn{4}{l}{ Overall Effect of therapy } \\
\cline { 2 - 4 } & \multicolumn{2}{|l|}{ TG I } & \multicolumn{1}{l|}{ TG II } \\
\hline Marked improvement $(>75 \%)$ & $f$ & $\%$ & $f$ & $\%$ \\
\hline Moderate improvement (>50-75\%) & 0 & 0 & 0 & 0 \\
\hline Mild improvement (>25-50\%) & 7 & $46.67 \%$ & 10 & $66.67 \%$ \\
\hline Unsatisfactory (<25\%) & 8 & $53.33 \%$ & 5 & $33.33 \%$ \\
\hline
\end{tabular}

\section{Observation}

In above table, the clinical effect of therapy shows in
TG I, 7 (46.67\%) patients were get Moderate improvement while $8(53.33 \%)$ patients were get Mild 
improvement. In TG II $10(66.67 \%)$ patients were get Moderate improvement while 5 (33.33\%) patients were get Mild improvement whereas no patients were there in Marked / Unsatisfactory improvement group in both groups.

\section{CONCLUSION}

The statistical adjudication with suitable parameters shows that both drugs (Vatari Guggulu \& Brihat Simhanada Guggulu) were extremely significant with pvalue $<0.0001$ on subjective $\&$ objective parameters among both groups in maximum cases.

The Clinical assessment of results shows that in TG I, 7 (46.67\%) patients were get Moderate improvement while $8(53.33 \%)$ patients were get Mild improvement. In TG II $10(66.67 \%)$ patients were get Moderate improvement while $5(33.33 \%)$ patients were get Mild improvement.

On comparing the effect of two therapies it can be concluded that TG II (Brihat Simhanada Guggulu) was more than TG I (Vatari Guggulu), but Mannwhitney test and unpaired $\mathrm{t}$ - test for comparison of effect of trial drugs between TGI and TGII shows statistically not significant that means the relief provided by each group was not so much differ.

\section{REFERENCES}

1. Bhawan A, Block B: Government of India Minis-try of AYUSH,. 2016;182.

2. Xu G, Zhou T, Gu Y, Wang Q, Shariff M, Gu P, et al. Evaluation of the Effect of Mega MSM on Improving Joint Function in Populations Experiencing Joint Degeneration. Int J Biomed Sci IJBS [Internet]. 2015 Jun

3. Cross M, Smith E, Hoy D, Carmona L, Wolfe F, Vos T, et al. The global burden of rheumatoid arthritis: estimates from the global burden of disease 2010 study. Ann Rheum Dis. 2014 Jul;73(7):1316-22.

4. Arthritis-India [Internet]. [cited 2021 Jan 3]. Available from: http://www.arthritis-india.com/rheumatoidarthritis.html

5. Management of Amavata (rheumatoid arthritis) with diet and Virechanakarma [Internet]. [cited 2021 Jan 3]. Available from: https://www.ncbi.nlm.nih.gov/pmc/articles/PMC50413

\section{Source of Support: Nil}

\section{Conflict of Interest: None Declared}

How to cite this URL: Praveen Kumar et al: The Management Of Amavata (Rheumatoid Arthritis) With Vatari Guggulu And Brihat Simhanada Guggulu. International Ayurvedic Medical Journal \{online\} 2021 cited February, 2021\} Available from: http://www.iamj.in/posts/images/upload/335 342.pdf 